Palavras chave:

Nutrição florestal

Malvaceae

Omissão de nutrientes

Sintomatologia

Histórico:

Recebido 02/08/2011

Aceito 22/10/2013

Keywords:

Forest nutrition

Malvaceae

Symptomatology

Omission of nutrients

Correspondência: camacho@uems.br
Marcos Antonio Camacho', Ana Paula Camara' , Andrei Rodriguez Zardin'

\section{DIAGNOSE VISUAL DE DEFICIENCIA DE NUTRIENTES EM MUDAS DE Bombacopsis glabra}

RESUMO: Bombacopsis glabra é uma espécie ornamental arbórea utilizada em programas de recuperação de áreas degradadas e composição paisagística. o conhecimento sintomatológico da deficiência nutricional apresentados por essa espécie possibilita a identificação e a correção em exemplares plantados. Neste trabalho, objetivou-se identificar sintomas visuais de deficiência nutricional em mudas de Bombacopsis glabra. $\bigcirc$ delineamento foi o inteiramente casualizado, com quatro repetições e nove tratamentos, sendo um completo e com omissão individual de N, P, K, Mg, S, Fe, B e Zn. Com exceção ao tratamento de omissão de $\mathrm{K}$, em todos os outros tratamentos foram evidenciados sintomas de deficiência nutricional. Dentre os sintomas diagnosticados, a clorose foi um sintoma característico nos tratamentos com omissão de $\mathrm{N}, \mathrm{Mg}, \mathrm{S}$ e $\mathrm{Zn}$, alternando apenas a intensidade e órgãos afetados. A omissão de boro foi caracterizada por folhas necrosadas e crestamento. A omissão de $\mathrm{K}$ provocou diminuição na massa seca das mudas, contudo as mesmas não manifestaram sintomas visuais, o que pode estar associado a um estoque de $\mathrm{K}$ na semente suficiente para o desenvolvimento inicial das mudas.

\section{VISUAL DIAGNOSIS OF DEFICIENCY OF NUTRIENTS IN Bombacopsis glabra SEDLINGS}

ABSTRACT: Bombacopsis glabra is an ornamental tree used to restore degraded areas and landscape composition. The nutritional deficiency symptoms presented by this species allows the identification and correction in growing plants. The objective of this study was to identify the visual symptoms of nutrient deficiency in seedlings of Bombacopsis glabra. The research was in completely randomized design with four repetitions and nine treatments, being a complete treatment (without omission of nutrients) and the other with the omission of $\mathrm{N}, \mathrm{P}, \mathrm{K}, \mathrm{Mg}, \mathrm{S}, \mathrm{Fe}, \mathrm{B}$ and $\mathrm{Zn}$. Excepting omission of the treatment with $\mathrm{K}$, in all others, it was observed symptoms. Among the diagnosed symptoms, chlorosis appeared as a characteristic symptom in the treatment of $\mathrm{N}, \mathrm{Mg}, \mathrm{S}$ and $\mathrm{Zn}$, only alternating the intensity and the affected organs. The omission of boron induced necrosis and blight on the leaves. The omission of $\mathrm{K}$ resulted in lower seedling dry mass; however they did not show visual symptoms, which may be associated with a stock of $K$ in the seed sufficient for early development of seedlings. 


\section{INTRODUÇÃO}

A espécie Bombacopsis glabra, conhecida como castanha-do-maranhão, castanha-da-praia, mamorana, cacao-selvagem, dentre outros nomes populares, é uma espécie ornamental arbórea da família Malvaceae, que mede de 4 a 6 m de altura (LORENZI, 1998; SCALON et al., 2003). Sua madeira é muito leve e mole, podendo ser usada para a confecção de objetos leves como caixotes, réguas e brinquedos. Sua distribuíção é comum na mata atlântica, sendo uma espécie nativa ocorrente no Estado de Mato Grosso do Sul (SCALON et al., 2003).

Há relatos na literatura sobre plantas desse gênero com possíveis ações anti-oxidantes para $B$. quitana (CALDERON et al., 2000) ou com propriedades fungicidas para B. glabra (PAULA et al., 2006).

Essa espécie pode ser utilizada tanto em programas de recuperação de áreas degradadas, como para composição paisagística e jardins. Em ambos os casos, o sucesso da utilização de espécies florestais está condicionada, dentre outros fatores, ao conhecimento da nutrição dessas espécies (SANGINGA et al., 1991). Segundo Carmo et al. (2006), plantas de Bombacopsis glabra possuem grande potencial para uso em sistemas silvipastoris, sendo utilizadas como cerca viva em algumas propriedades rurais e em arborização.

No Brasil, tem sido desenvolvidos diversos trabalhos sobre nutrição de espécies florestais, entretanto, esses estudos ainda são incipientes, uma vez que abrangem apenas uma parte das espécies existentes nos biomas brasileiros. Uma das formas usuais para diagnóstico a campo é a diagnose visual (MALAVOLTA, 2006), entretanto, relatos para espécies florestais são ainda mais escassos.

Destacam-se alguns trabalhos desenvolvidos com sintomatologia de nutrientes em espécies florestais: teca (Tectona grandis) (BARROSO et al., 2005), jatobá (Hymenaea courbaril var. stilbocarpa) (MENDONÇA et al., 1999), paricá (Schizolobium amazonicum) (MARQUES et al., 2004), mogno (Swietenia macrophilla King) (SILVA et al., 2007; WALLAU et al., 2008) e sangra d'água (Croton urucurana) (SORREANO et al. 2008).

Renó et al. (1997) relataram que a produção de massa seca da parte aérea e da raiz foram prejudicados nos tratamentos com deficiência de $\mathrm{N}, \mathrm{P}$ e $\mathrm{S}$ para canafístula (Senna multijuga), cedro (Cedrela fissilis), pau-ferro (Caesalpinea ferrea var. leiostachya) e jacaré (Piptadenia gonoacantha), concluindo que solos com baixa capacidade de fornecer esses nutrientes seriam limitantes ao desenvolvimento dessas espécies.
Face ao exposto, neste trabalho, objetivou-se identificar possíveis sintomas de deficiência de nutrientes em mudas de Bombacopsis glabra.

\section{MATERIAL E MÉTODOS}

O experimento foi conduzido em condições de casa de vegetação na UEMS (Universidade Estadual de Mato Grosso do Sul) - Unidade Universitária de Aquidauana. Foram utilizadas plantas de castanheira do maranhão (Bombacopisis glabra) oriundas de sementes obtidas na região do ecótono Cerrado/Pantanal.

As sementes foram germinadas em bandejas contendo vermiculita, umedecidas diariamente, sendo que 12 dias após a emergência (por ocasião do aparecimento da primeira folha verdadeira) as plântulas foram transplantadas para vasos com capacidade de $5 \mathrm{~L}$, contendo areia lavada. Após os transplantes, as plantas receberam uma solução nutritiva com I/5 da concentração da solução de Johnson et al. (1957), modificada. Aos 30 dias após o transplante, iniciou-se a aplicação dos tratamentos.

O delineamento experimental foi o inteiramente casualizado, com nove tratamentos - completo, omissão de $\mathrm{N}$, omissão de $\mathrm{P}$, omissão de $\mathrm{K}$, omissão de $\mathrm{Mg}$, omissão de $\mathrm{S}$, omissão de $\mathrm{B}$, omissão de $\mathrm{Fe}$ e omissão de $\mathrm{Zn}$ - e quatro repetições. Cada planta foi considerada uma unidade experimental.

A solução nutritiva completa apresentou a seguinte composição de acordo com Johnson et al. (1957): $6,0 \mathrm{~mL}$ de $\mathrm{KNO}_{3} \mathrm{I} \mathrm{mol} \cdot \mathrm{L}^{-1} ; 4,0 \mathrm{~mL}$ de $\mathrm{Ca}\left(\mathrm{NO}_{3}\right) \cdot 4 \mathrm{H}_{2} \mathrm{O} \mathrm{I} \mathrm{mol} \cdot \mathrm{L}^{-1} ; 2,0 \mathrm{~mL}$ de $\mathrm{NH}_{4} \mathrm{H}_{2} \mathrm{PO}_{4} \mathrm{I} \mathrm{mol} \cdot \mathrm{L}^{-1}$; I, $0 \mathrm{~mL}$ de $\mathrm{MgSO}_{4} \cdot 7 \mathrm{H}_{2} \mathrm{O}$ I mol. $\mathrm{L}^{-1} ; \mathrm{I}, 0 \mathrm{~mL}$ de Fe-EDTA $0,2 \mathrm{~mol} \cdot \mathrm{L}^{-1} ; \mathrm{I}, 0 \mathrm{~mL}$ de $\mathrm{KCl} 0,05 \mathrm{~mol} \cdot \mathrm{L}^{-1} ; \mathrm{I}, 0 \mathrm{~mL}$ de $\mathrm{H}_{3} \mathrm{BO}_{3}$ $0,02 \mathrm{~mol} \cdot \mathrm{L}^{-1} ; \mathrm{I}, 0 \mathrm{~mL}$ de $\mathrm{MnSO}_{4} \cdot \mathrm{H}_{2} \mathrm{O} 0,002 \mathrm{~mol} \cdot \mathrm{L}^{-1}$; $1,0 \mathrm{~mL}$ de $\mathrm{ZnSO}_{4} \cdot 7 \mathrm{H}_{2} \mathrm{O} \quad 0,002 \mathrm{~mol} \cdot \mathrm{L}^{-1} ; 1,0 \mathrm{~mL}$ $\mathrm{CuSO}_{4} \cdot 5 \mathrm{H}_{2} \mathrm{O} \quad 0,0005 \mathrm{~mol} \cdot \mathrm{L}^{-1} ; \quad 1,0 \mathrm{~mL} \mathrm{H}_{2} \mathrm{MoO}_{4}(85 \%$ $\left.\mathrm{MoO}_{3}\right)$ 0,0005 mol. $\mathrm{L}^{-1}$.

$\mathrm{Na}$ solução dos tratamentos com omissão de nutrientes, as concentrações foram idênticas à solução completa, exceto quanto ao nutriente omitido.

As mudas permaneceram na casa de vegetação até os 120 dias após o início dos tratamentos, com altura de $20 \mathrm{~cm}$, quando foram colhidas, separandoas em parte aérea (folhas + ramos + caule) e raízes, as quais foram lavadas em água deionizada e secas em estufa de circulação forçada de ar, com temperatura variando de $65^{\circ} \mathrm{C}$ a $70^{\circ} \mathrm{C}$, até atingir massa constante. Após a secagem, foi determinada a massa da matéria seca da parte aérea (MSPA) (folhas + ramos + caule), raiz (MSR) e total (MST). 
Os dados foram submetidos à análise de variância, sendo adotada a equação $I$, sendo $Y_{i j}=a$ unidade experimental que recebeu o i-ésimo efeito do tratamento (omissões de nutrientes) na j-ésima repetição; $\mu=$ média geral; $\mathrm{T}_{\mathrm{i}}=$ efeito do i-ésimo tratamento; e $\varepsilon \mathrm{ij}$ $=$ efeito do erro aleatório.

$$
Y_{i j}=\mu+T_{i}+\varepsilon_{i j}
$$

As médias dos tratamentos foram comparadas pelo teste $t$ (LSD) ao nível de $5 \%$ de probabilidade.

\section{RESULTADOS E DISCUSSÃO}

A produção de MST foi influenciada negativamente quando as plantas foram submetidas à omissão de $\mathrm{N}, \mathrm{K}, \mathrm{S}$ e B, causando diminuições de até $30 \%$. A produção de MSR foi influenciada pela omissão de N, P, K, Mg, S e B (Tabela I), sendo evidenciada a diminuição da MSR quando da submissão das plantas aos tratamentos que não receberam esses nutrientes. Essa resposta diverge do obtido por Duboc et al. (1996), estudando o jatobá (Hymenaea courbaril L. var. stilbocarpa), e Wallau et al. (2008), em estudos com mogno (Swietenia macrophilla), mas se assemelha aos resultados encontrados por Mendonça et al. (1999), para aroeira-do-sertão (Myracrodruon urundeuva), que observaram menor produção de MSR nos tratamentos com omissão de $\mathrm{P}, \mathrm{K}, \mathrm{Mg}$ e $\mathrm{B}$.

TABELA I Massa seca da raiz (MSR), da parte aérea (MSPA) e total (MST) de plantas de Bombacopsis glabra submetidas a deficiência de nutrientes.

TABLE I Root (MSR), shoot (MSPA) and total biomass (MST) of Bombacapsis glabra under nutrient deficiency.

\begin{tabular}{cccc}
\hline Tratamentos & MSR (g) & MSPA (g) & MST (g) \\
\hline Completo & $4,05 \mathrm{a}$ & $2,99 \mathrm{ab}$ & $7,04 \mathrm{ab}$ \\
$-\mathrm{N}$ & $2,87 \mathrm{~b}$ & $1,94 \mathrm{c}$ & $4,8 \mathrm{I} \mathrm{e}$ \\
$-\mathrm{P}$ & $2,99 \mathrm{~b}$ & $3,45 \mathrm{ab}$ & $6,44 \mathrm{bcd}$ \\
$-\mathrm{K}$ & $2,66 \mathrm{~b}$ & $2,77 \mathrm{abc}$ & $5,43 \mathrm{cde}$ \\
$-\mathrm{Mg}$ & $3,02 \mathrm{~b}$ & $2,72 \mathrm{abc}$ & $5,75 \mathrm{bcde}$ \\
$-\mathrm{S}$ & $2,79 \mathrm{~b}$ & $2,66 \mathrm{bc}$ & $5,45 \mathrm{cde}$ \\
$-\mathrm{B}$ & $3,02 \mathrm{~b}$ & $2,13 \mathrm{c}$ & $5,16 \mathrm{de}$ \\
- Fe & $4,20 \mathrm{a}$ & $3,5 \mathrm{I} \mathrm{a}$ & $7,7 \mathrm{I} a$ \\
- Zn & $3,38 \mathrm{ab}$ & $3,1 \mathrm{I} \mathrm{ab}$ & $6,49 \mathrm{abc}$ \\
\hline $\mathrm{CV}(\%)$ & 18,67 & 20,83 & 14,88 \\
\hline
\end{tabular}

MSR - massa seca da raiz;

MSPA - massa seca da parte aérea;

MST - massa seca total
$\mathrm{Na}$ avaliação da parte aérea das plantas, apenas os tratamentos com deficiência de $\mathrm{N}$ e $\mathrm{B}$ ocasionaram diminuição na produção MSPA (Tabela I). Essa resposta às omissões de $\mathrm{Ne} \mathrm{B}$ não foram relatadas para $\mathrm{H}$. courbaril (DUBOC et al., 1996), enquanto Mendonça et al. (1999) também relataram essas diminuições nas omissões desses nutrientes em M. urundeuva. Barroso et al. (2005) relataram redução na produção MSPA em todos os tratamentos com deficiência de macronutrientes, sendo que o macronutriente que provocou a menor produção de biomassa foi o N. Sorreano et al. (2008), trabalhando com deficiência de micronutrientes em sangra d'água (Croton urucurana), não encontram diferenças na produção de MSPA.

A omissão de $\mathrm{N}$ em mudas de Bombacopsis glabra resultou na clorose das folhas mais velhas, evoluindo para uma clorose generalizada e início de clorose nas folhas mais novas (Figura la). Esses mesmos sintomas foram relatados para diversas espécies florestais, como $M$. urundeuva (MENDONÇA et al., 1999), Tectona grandis (BARROSO et al., 2005), Cedrella fissilis (SILVA; MUNIZ, 1995), Schizolobium amazonicum (MARQUES et al., 2004) e Swietenia macrophilla (WALLAU et al., 2008). A clorose é resultado de diminuição da síntese de clorofila (MALAVOLTA, 2006), e nos cloroplastos, segundo relatado para Hazah e Gomes (1979) para seringueira, ocorre a desorganização das pilhas de tilacóides, bem como do aumento dos grânulos de amido no interior dos cloroplastos. $O$ sistema radicular teve o crescimento reduzido (Figura I b), o que corrobora com o relatado de Barroso et al. (2005).
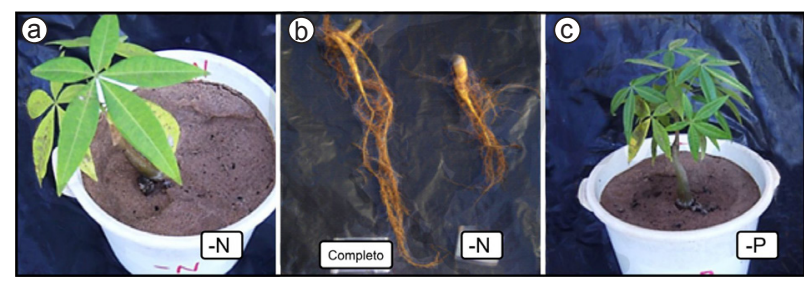

FIGURA I Sintomas de deficiência de nitrogênio (a,b) e fósforo (c) em mudas de Bombacopsis glabra.

FIGURE I Deficiency symptoms of nitrogen $(\mathrm{a}, \mathrm{b})$ and phosphorus (c) in Bombacopsis glabra seedlings.

$\mathrm{Na}$ omissão de fósforo, observou-se maior número de brotações que as demais e sua coloração oscilou de verde escuro para tons menos fortes nas folhas mais velhas (Figura Ic). Mendonça et al. (1999) em trabalho com mudas de $M$. urundeuva encontraram sintomas folhas mais velhas com aspecto arroxeado, o que se assemelha com o observado neste trabalho, entretanto para Cedrella fissilis, Silva e Muniz (1995) 
relataram escurecimento apenas das nervuras. Em Tectona grandis, Barroso et al. (2005) observaram encarquilhamento nas extremidades das folhas mais velhas e, em contraposição ao aparecimento de folhas mais escuras, clorose nas folhas mais novas.

Não se verificaram sintomas de deficiência de K, o que pode ser indicativo de que haja alto estoque desse nutriente na semente e/ou, ainda, boa capacidade da planta em utilizar $\mathrm{K}$, conforme evidenciado para outras espécies vegetais (WHITE et al., 20 I0), ou ainda pode ser indicativo de uma alta capacidade da planta em utilizar K, ou seja, possui uma alta eficiência no uso desse nutriente para período de avaliação estudado. Em condições de deficiência de $K$ são relatados enrugamento das folhas que, posteriormente, apresentavam necrose em plantas de M. urundeuva (MENDONÇA et al., 1999), clorose internerval e pontos necróticos em folhas de Tectona grandis (BARROSO et al., 2005) e manchas cloróticas que evoluiram para necroses nas proximidades marginais das folhas mais velhas de Schizolobium amazonicum (MARQUES et al., 2004).

Nas mudas submetidas à deficiência de $\mathrm{Mg}$ verificou-se nas folhas mais velhas uma ligeira clorose internerval e manchas foliares (Figura 2a). Esses mesmos sintomas foram observados em $M$. urundeuva (MENDONÇA et al., 1999), Tectona grandis (BARROSO et al., 2005) e Schizolobium amazonicum (MARQUES et al., 2004). Essa clorose está ligada a síntese de clorofila, uma vez que o $\mathrm{Mg}$ faz parte dessa molécula.

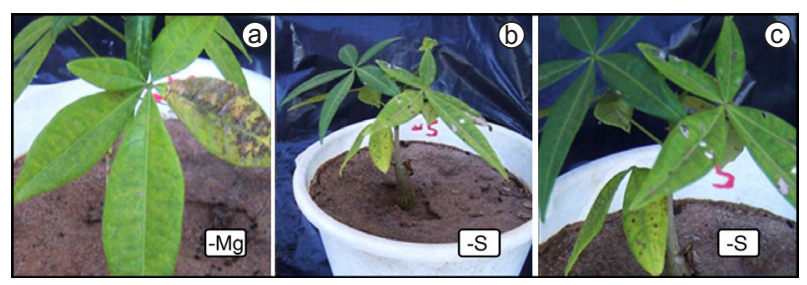

FIGURA 2 Sintomas de deficiência de magnésio (a) e enxofre (b,c) em mudas de Bombacopsis glabra.

FIGURE 2 Deficiency symptoms of magnesium (a) and sulfur $(b, c)$ in Bombacopsis glabra seedlings.

Em condições de deficiência de $S$, observaramse manchas foliares, evoluindo para clorose (Figura $2 b$ e $2 c$ ). Marques et al. (2004) encontraram sintomas parecidos para Schizolobium amazonicum, enquanto Mendonça et al. (1999) não evidenciaram qualquer alteração para $M$. urundeuva nos tratamentos deficientes em S.

A deficiência de $B$ foi evidenciada por folhas necrosadas (Figura 3a), crestamento (requeima), sintomas estes semelhantes aos encontrados por
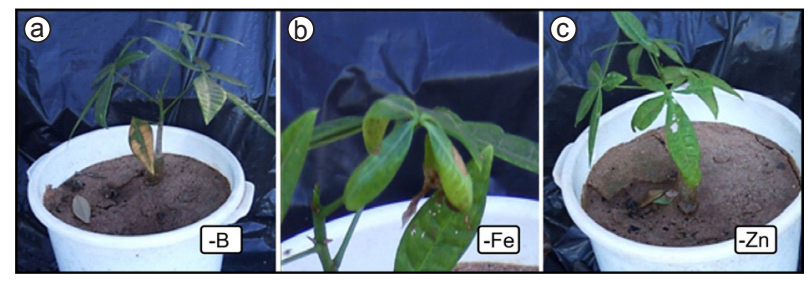

FIGURA 3 Sintomas de deficiência de boro (a), ferro (b) e zinco (c) em mudas de Bombacopsis glabra.

FIGURE 3 Deficiency symptoms of boron (a), iron (b) and zinc (c) in Bombacopsis glabra seedlings.

Sorreano et al. (2008) para Croton urucurana, e reduzido desenvolvimento caulinar (Tabela I). O crescimento caulinar reduzido também foi observado por Marques et al. (2004) para Schizolobium amazonicum, evidenciado pelo aparecimento de internódios foram mais curtos. Em outras espécies florestais também são relatadas a morte do meristema apical da planta (MARQUES et al., 2004; MENDONÇA et al., 1999; SORREANO et al., 2008), provocando perda da dominância apical com subsequentes emissões de gemas axilares, embora não tenha sido verificado este sintoma para B. glabra.

Nas mudas sob omissão de $\mathrm{Fe}$ foram evidenciadas manchas cloróticas e crescimento reduzido, e nas folhas mais jovems ocorreu encurvamento (Figura 3b) e amarelecimento do limbo foliar. Esses sintomas estão de acordo com Mendonça et al. (1999) e Sorreano et al. (2008), entretanto divergem dos relatados por Marques et al. (2004) em estudos com Schizolobium amazonicum, que foram caracterizados como clorose generalizada e intensa, tanto nas folhas jovens quanto nas folhas velhas. A clorose provocada pela deficiência de Fe foi, provavelmente, causada por baixa produção de clorofila (MALAVOLTA et al., 1997).

Em mudas sob a omissão de $\mathrm{Zn}$ verificou-se encurtamento de internódios, com folhas bem próximas e clorose no limbo foliar (Figura 3c). Esses sintomas são semelhantes aos observados para Schizolobium amazonicum (MARQUES et al., 2004) e Croton urucurana (SORREANO et al., 2008). A deficiência de Zn muitas vezes não é encontrada em plantas, porque em razão da baixa quantidade necessária desse micronutriente para algumas espécies, o conteúdo da semente pode colaborar para o suprimento inicial desse nutriente (LANGE et al., 2005).

\section{CONCLUSÕES}

A omissão de $\mathrm{N}, \mathrm{B}, \mathrm{K}, \mathrm{S}$ e $\mathrm{Mg}$ reduzem a produção de massa seca da planta em mudas de Bombacopsis glabra. 
Na omissão de N, P, Mg, B, Fe e Zn observaramse sintomas semelhantes aos descritos para outras espécies florestais. $\mathrm{E}$ para $\circ \mathrm{K}$ não foram observados sintomas característicos.

\section{AGRADECIMENTOS}

Ao Programa Institucional de Bolsas de Iniciação Científica da Universidade Estadual de Mato Grosso do Sul (PIBIC/UEMS), pela concessão de bolsa para o segundo autor.

\section{REFERÊNCIAS}

BARROSO, D. G.; FIGUEIREDO, F. A. M. M. A.; PEREIRA, R. C.; MENDONÇA, A. V. R.; SILVA, L. C. Diagnóstico de deficiências de macronutrientes em mudas de teca. Revista Árvore, Viçosa, v. 29, n. 5, p. 67I-679, 2005.

CALDERON, A. I.; ANGERHOFER, C. K.; PEZZUTO, J M.; FARNSWORTH, N. R.; FOSTER, R.; CONDIT, R.; GUPTA, M. P.; SOEJARTO, D. D. Forest plot as a tool to demonstrate the pharmaceutical potencial of plants in a tropical forest of Panama. Economic Botany, New York, v. 54, n. 3, p. 278-294, 2000.

CARMO, V. B.; REBOLLAR, P. M.; MILLER, P. R. Utilização da castanheira da praia (Bombacopsis glabra) como mourão vivo pelas comunidades açorianas da ilha de Santa Catarina. In: REUNIÃO ANUAL DA SBPC, 58., 2006, Florianópolis. Anais... Florianópolis: UFSC, 2006. I CD-ROM.

DUBOC, E.; VENTORIM, N.; VALE, F. R.; DAVIDE, A. C. Nutrição do jatobá (Hymenaea courbaril L. var. stilbocarpa (Hayne) Lee et Lang.). Cerne, Lavras, v. 2, n. I, p. I38I52, 1996.

HAMZAH, S. B.; GOMEZ, J. B. Ultrastructure of mineral deficient leaves of Hevea I. effects of macronutrients deficiencies. Journal of the Rubber Research Institute of Malaysia, Kuala Lumpur, v. 27, n. 3, p. I32-142, 1979.

JOHNSON, C. M.; STONTS, P. R.; BROGER, T. C.; CARLTON, A. B. Comparative chlorine requirements of different plants species. Plant and Soil, The Hague, v. 8, p. 337-353, 1957.

LANGE, A.; MARTINES, A. M.; SILVA, M. A. C. da; SORREANO, M. C. M.; CABRAL, C. P.; MALAVOLTA, E. Efeito de deficiência de micronutrientes no estado nutricional da mamoneira cultivar Íris. Pesquisa Agropecuária Brasileira, Brasília, v. 40, n. I, p. 6I-67, jan. 2005

LORENZI, H. Árvores brasileiras: manual e cultivo de plantas arbóreas nativas do Brasil. Nova Odessa: Plantarum, 1998. $352 \mathrm{p}$.

MALAVOLTA, E. Manual de nutrição mineral de plantas. São Paulo: Ceres, 2006. 638 p.
MALAVOLTA, E.; VITTI, G. C.; OLIVEIRA, S. A. Avaliação do estado nutricional das plantas: princípios e aplicações. 2. ed. Piracicaba: POTAFOS, 1997. 319 p.

MARQUES, T. C. L. L. S. M.; CARVALHO, J. G.; LACERDA, M. P. C.; MOTA, P. E. F. Crescimento inicial do paricá (Schizolobium amazonicum) sob omissão de nutrientes e de sódio em solução nutritive. Cerne, Lavras, v. 10, n. 2, p. I84-195, 2004

MENDONÇA, A. V. R.; NOGUEIRA, F. D.; VENTURIN, N.; SOUZA, J. S. Exigências nutricionais de Myracrodruon urundeuva Fr. All (aroeira do sertão). Cerne, Lavras, v. 5, n. 2, p. $65-75,1999$.

PAULA, V. F.; CRUZ, M. P.; BARBOSA, L. C. A. Constituintes químicos de Bombacopsis glabra (Bombacaceae). Química Nova, São Paulo, v. 29, n. 2, p. 2/3-2।5, 2006.

RENÓ, N. B.; SIQUEIRA, J. O.; CURI, N.; VALE, F. R. Limitações nutricionais ao crescimento inicial de quatro espécies arbóreas nativas em Latossolo Vermelho-Amarelo. Pesquisa Agropecuária Brasileira, Brasília, v. 32, n. I, p. 17-25, jan. 1997.

SANGINGA, N.; GWAJE, D.; SWIFT, M. J. Nutrient requeriments of exotic tree species in Zimbabwe. Plant and Soil, The Hague, v. I32, p. 197-205, I991.

SCALON, S. P. Q.; MUSSURY, R. M.; RIGONI, M. R.; SCALON FILHO, H. Crescimento inicial de mudas de Bombacopsis glabra (Pasq.) A. Robyns sob condições de sombreamento. Revista Árvore, Viçosa, v. 27, n. 6, p. 753-758, 2003.

SILVA, M. A. G.; MUNIZ, A. S. Exigências nutricionais de cedro (Cedrela fissilis Velloso) em solução nutritiva. Revista Árvore, Viçosa, v. 19, n. 3, p. 415-425, 1995.

SILVA, W. G.; TUCCI, C. A. F.; HARA, F. A. S.; SANTOS, R. A. C. Efeito de micronutrientes sobre o crescimento de mudas de mogno (Swietenia macrophilla King) em Latossolo amarelo. Acta Amazonica, Manaus, v. 37, n. 3, p. 37I376, 2007.

SORREANO, M. C. M.; MALAVOLTA, E.; SILVA, D. H.; CABRAL, C. P.; RODRIGUES, R. R. Deficiência de micronutrientes em mudas de sangra d'água (Croton urucurana, Baill). Cerne, Lavras, v. 14, n. 2, p. 126-132, 2008.

WALLAU, R. L. R.; BORGES, A. R.; ALMEIDA, D. R. A.; CAMARGOS, S. L. Sintomas de deficiências nutricionais em mudas de mogno cultivadas em solução nutritiva. Cerne, Lavras, v. I4, n. 4, p. 304-310, 2008.

WHITE, P. J.; HAMMOND, J. P.; KING, G. J.; BOWEN, H. C.; HAYDEN, R. M.; MEACHAM, M. C.; SPRACKLEN, W. P.; BROADLEY, M. R. Genetic analysis of potassium use efficiency in Brassica oleracea. Annals of Botany, London, v. 105, p. I199-1210, 2010. 
